\title{
Role of Whole-Body Tc 99m MDP Bone Scintigraphy for Evaluating Skeletal Metastasis in Patients with Lung Cancer
}

\author{
Afrin $\mathrm{R}^{1}$, Haque $\mathrm{FS}^{1}$, Biswas $\mathrm{SK}^{1}$, Hossain $\mathrm{S}^{1}$, Roy $\mathrm{SK}^{2}$, Jahan $\mathrm{M}^{3}$ \\ ${ }^{1}$ Institute of Nuclear Medicine \& Allied Sciences, DMCH Campus, Bangladesh \\ ${ }^{2}$ Department of Radiology Imaging, SSMC \& Mitford Hospital, Dhaka, Bangladesh \\ ${ }^{3}$ Consultant Radiologist \\ e-mail:afrinrbd@gmail.com
}

\begin{abstract}
Lung cancer is the third most common site of origin of metastatic cancer deposits in bone, after breast and prostate. It's metastasis to bone is one of the most aggressive tumors and has a very unfavorable prognosis. This retrospective descriptive study was designed to detect the skeletal metastasis of carcinoma (Ca) lung patient by Tc $99 \mathrm{~m}$ MDP bone scan. The medical records of all patients attended between January 2015 and July 2015 with a diagnosis of lung cancer were reviewed. Lung cancer in all patients was confirmed pathologically, and patients underwent whole-body bone scan for evaluating skeletal metastasis. Patient with clinical and laboratory evidence of infection, trauma, metabolic disease or arthropathy were not included in the study. Bone scan was done after three hours of intravenous administration of 20mci Tc 99m MDP (methylene diphosphonate) and images were obtained on a gamma camera. The mean age of the patients was Mean \pm SD was $55.5 \pm 12.5$ with range from 31 to 90 years. Out of 47 cases, 36 (76.59\%) were diagnosed as positive for skeletal metastasis by bone scan and 11 (23.41\%) were negative for bony metastasis. Among 36 positive patients, 28 patients (77.86\%) were histopathologically diagnosed as adenocarcinoma, 7 patients $(19.44 \%)$ had squamous cell carcinoma and only one patient $(2,7 \%)$ had small cell carcinoma. Bone scan findings were compared by either conventional X-ray/CT scan/MRI /pathologically. In present study, the distribution of lesions in bone scan had recorded. Maximum 47.22\% lesions were found in ribs, $27.77 \%$ lesions were in lumbar vertebrae, $19.44 \%$ in thoracic vertebrae, $19.44 \%$ in joints, $16.66 \%$ in long bones (femur and humerus), $11.11 \%$ in skull bones, $22.22 \%$ in pelvic bones, $5.55 \%$ in clavicle and $2.77 \%$ in scapula. Tc $99 \mathrm{~m}$ MDP bone scan plays a pivotal role for detection of skeletal metastasis which is very essential to manage Ca lung patient. As bone scintigraphy is very cost effective in govt. nuclear medicine centre in comparison to other imaging modalities, so it can play a major role in detecting skeletal metastasis in ca lung patients in a developing country like Bangladesh.
\end{abstract}

Key words: bone scan, Ca lung, Tc 99m MDP, bone metastases.

\section{Introduction}

Lung cancer is the second most common cancer and the leading cause of cancer related mortality in both male and female. Lung cancer is the third most common site of origin of metastatic cancer deposits in bone.

Involvement of bone in lung cancer metastases is a major source of morbidity and mortality arising from skeletal related events (SREs). Common complications include intractable pain, bone destruction, hypercalcemia, nerve compression syndromes, and pathological fractures. ${ }^{1}$

Imaging has an important role in the detection, diagnosis, prognostication, treatment planning, and follow-up monitoring of bone metastases. Tc $99 \mathrm{~m}$ MDP bone scintiscanning is widely regarded as the most cost-effective and available whole-body screening test for the assessment of bone metastases. Tc 99m diphosphonates most commonly Tc $99 \mathrm{~m}$ methylene diphosphonate (MDP) is the most frequently used isotope. 
Indications for bone scintiscanning include; staging in asymptomatic patients, evaluating persistent pain in the presence of equivocal or negative radiographic findings, determining the extent of bone metastases in patients with positive radiograph findings, differentiating metastatic from traumatic fractures by assessing the pattern of involvement, determining the therapeutic response to metastases. ${ }^{2}$

Isotopic imaging methods depict bone metastatic lesions as areas of increased tracer uptake. The classic pattern appears as the presence of multiple randomly distributed focal lesions throughout the skeleton, may be a solitary scintigraphic abnormality or just a few lesions. Other patterns include diffuse involvement (super scan), photopenic lesions (cold lesions), normal scintiscans, flare phenomena, and softtissue lesions. $^{2}$

Conventional radiography is the preferred modality for characterizing lesions that are depicted on bone scintiscans. However radiographs are relatively insensitive in the detection of early or small metastatic lesions. Although CT scans are superior to radiographs, CT scanning is also relatively insensitive in showing small intramedullary lesions. FDG-PET scanning has limited spatial resolution, and complementary CT scanning or MRI is required to localize an area of increased glucose metabolism. In skull metastases, the high rate of glucose metabolism in the normal areas of brain may obscure metastases. Whole-body MRI and FDG-PET scanning is excellent in detection of skeletal metastases, but it is not widely available in the country. Moreover it is very expensive.

This retrospective descriptive study was designed to detect the skeletal metastasis of carcinoma (Ca) lung patient by Tc 99m MDP bone scan.

\section{Materials and Methods}

The medical records at the Institute of Nuclear Medicine \& Allied Sciences (INMAS), Dhaka, were reviewed retrospectively to identify all patients attended during January-July 2015 with a lung cancer. Lung cancer in all patients was confirmed histopathologically, and patients underwent whole-body scintigraphy for evaluation of skeletal metastases.

All patients' records were reviewed retrospectively and imaging reports. Patient with clinical and laboratory evidence of infection, trauma, metabolic disease or arthropathy were not included in the study. A total of 47 patients met the inclusion criteria. Bone scan (BS) was performed by the intravenous administration of technetium $99 \mathrm{~m}$ methylene diphosphonate (MDP) at a dose of $20 \mathrm{mCi}$. Images were obtained on a dual head gamma camera (Siemens Health Care System). Bone phase images were taken approximately three hrs after injection of the radiotracer and the scan time is about 15 minutes for a whole body scan.

Two experienced nuclear medicine physicians interpreted the bone scans as positive for bone metastasis if they contained one or more abnormal focal increased uptake sites.

Bone scan findings were compared with other imaging modalities, such as simple radiography (local X-ray), Computed Tomography (CT), Magnetic Resonance Imaging (MRI) or with histopathology findings (Image guided biopsy).

\section{Results}

The mean age of the patients was Mean \pm SD was $55.5 \pm 12.5$ with range from 31 to 90 years. Of the total 47 patients, $38(80 \%)$ were male and nine $(20 \%)$ were female. Out of 47 cases, 36 $(77 \%)$ were diagnosed as positive for skeletal metastasis by bone scan and 11 (23\%) were negative for bony metastasis.

Table I: Distribution of study patients by skeletal metastases

\begin{tabular}{lcc}
\hline $\begin{array}{l}\text { Skeletal } \\
\text { metastases }\end{array}$ & Frequency & Percentage \\
\hline Positive & 36 & 77 \\
Negative & 11 & 23 \\
Total & $\mathbf{4 7}$ & $\mathbf{1 0 0 . 0}$ \\
\hline
\end{tabular}


Among the 36 patients with positive skeletal metastasis, maximum were non small cell lung cancer (NSCLC), 28 patients (78\%) were histopathologically diagnosed as adenocarcinoma, seven (19\%) patients had squamous cell carcinoma and only one patient (3\%) had small cell carcinoma. (figure1)

Figure 1: Distribution of the bone scan positive patients, according to histopathological types: $(n=36)$

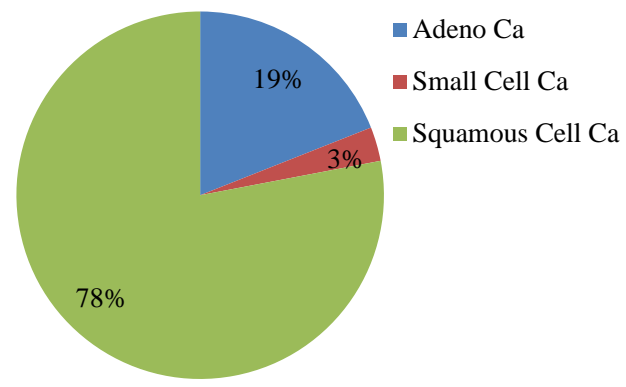

Of the 36 patients, 28 (78\%) had multiple infiltrative lesions in bone scan and eight (22\%) had solitary lesion, and $20 \quad(56 \%)$ had characteristic bone pain, but 16 patients (44\%) were asymptomatic.

Table II: Distribution of bone scan positive patients by characteristic bone pain was

\begin{tabular}{lcc}
\hline Bone pain & Frequency & Percentage \\
\hline Positive & 20 & 56 \\
Negative & 16 & 44 \\
Total & $\mathbf{3 6}$ & $\mathbf{1 0 0 . 0}$ \\
\hline
\end{tabular}

Twenty tow patients $(61 \%)$ received both chemotherapy (CT) and radiotherapy (RT), three patients $(8 \%)$ were underwent surgery and received chemotherapy also. Eleven patients $(31 \%)$ were newly diagnosed, and had no treatment history (figure 2).

Serum alkaline phosphatase was elevated in 16 patients, among 36 positive patients. In present study, the distribution of lesions in bone scan had recorded.
Figure 2: Distribution of the bone scan positive patients, according to treatment status: $(\mathrm{n}=36)$

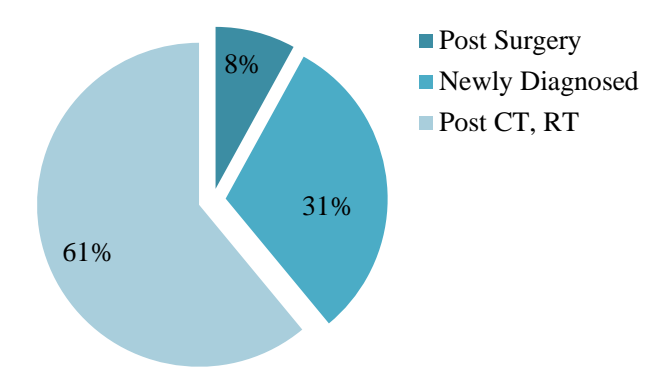

Serum alkaline phosphatase was elevated in 16 patients, among 36 positive patients. In present study, the distribution of lesions in bone scan had recorded.

Maximum lesions were found on axial skeleton. $47 \%$ lesions were found in ribs, $27 \%$ lesions were in lumbar vertebrae, $20 \%$ lesions were found in thoracic vertebrae, $20 \%$ found in joints, $16 \%$ found in long bones (femur and humerus), $11 \%$ in skull bones, $22 \%$ in pelvic bones, $5 \%$ in clavicle and $3 \%$ lesions were found in scapula. (Figure 3).

Figure 3: Distribution of the metastatic lesions according to anatomic locations

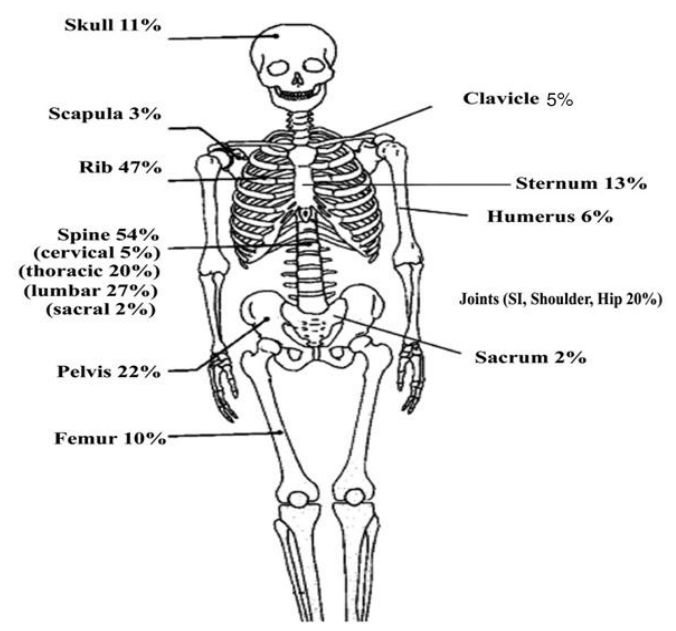

One scan findings were compared with other imaging modalities, such as simple radiography (local X-ray), Computed Tomography (CT), 
Magnetic Resonance Imaging (MRI) or with histopathology findings (Image guided biopsy). Out of 36 patients, 20 have CT guided biopsy reports and all 20 patients were diagnosed as positive for bone metastasis in pathological test also.

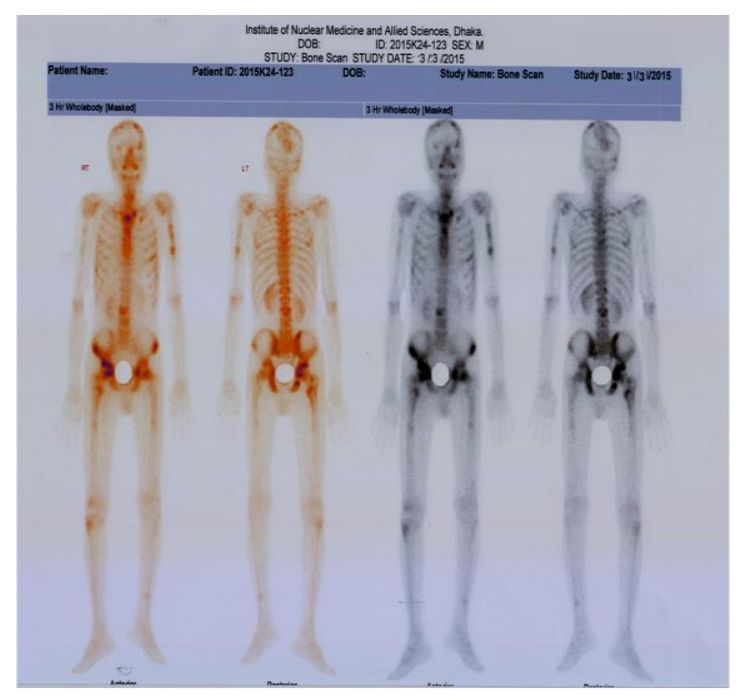

Figure 4:

- 50 years male, diagnosed case of adenocarcinoma right lung, no history of surgery and radiotherapy, chemotherapy was ongoing , 2 cycles were given.

- Bone scintiscan findings: Multiple infiltrative lesions on both frontal and left parietal region, upper shaft of left humerus, manubrium sterni including left sternoclavicular joint, left 2nd, 6th, right 7th rib anteriorly, right 3rd rib posteriorly,L3 vertebra, both pubic bones, both acetabulum, trochantaric region of right femur.

- Bone scan findings compared with local $\mathrm{X}$-ray findings and findings were compatible. CT guided needle biopsy done from L3 vertebral body showed metastatic adenocarcinoma

\section{Discussion}

Lung cancer is the most common neoplasm worldwide and the bone is one of the most common metastatic sites for lung cancer. ${ }^{5,6}$

Non-small cell lung cancer (NSCLC) accounts for $80-85 \%$ of all lung cancer diagnoses, the majority of which present as late-stage disease, primarily owing to the asymptomatic nature of early disease and commonly metastasize to bone. In this study, maximum 28 patients (78\%) with positive scintigraphy report were histopathologically diagnosed as adenocarcinoma, study showed that adenocarcinoma was the most common cell type found in patients with bone metastases, in their study. ${ }^{7}$

In the present study, high incidence of bone metastasis from lung cancer was seen in the axial skeleton like vertebra, rib, and pelvis. The distribution of the lesions are similar to the previous studies. ${ }^{8}$ In bone metastasis from lung cancer, metastasis may occur easily at an axial bone through the vertebral vein system at an early stage and then at an appendicular bone in more advanced stages of the disease. The prognosis is poorer in patients with metastasis to the appendicular bone. ${ }^{8}$

Ebert et al observed that bone metastasis was present in $20-30 \%$ of patients at the initial diagnosis of lung cancer in their study, which strongly supports the findings of present study. ${ }^{9}$ In present study, out of 36 bone scan positive patients, $20(56 \%)$ had characteristic bone pain, but 16 patients $(44 \%)$ were asymptomatic. Serum alkaline phosphatase level was elevated in 16 among 36 positive patients. Iordanidou $\mathrm{L}$ et al found significant number of clinically asymptomatic patients diagnosed as positive for metastases on scintigraph. ${ }^{10}$ Other studies reported that the prevalence of bone metastasis among patients who report bone pain was almost similar to patients who did not report bone pain.

Bone metastasis is a major determinant of treatment outcome, quality of life, and survival for lung cancer patients. The mean length of survival is substantially longer in patients with solitary-site metastasis than in patients with multiple-site metastases. ${ }^{11}$

Joo-Won Min et al suggested in their study that in patients with disseminated disease who do not need evaluation of nodal staging, BS and the measurement of serum ALP concentration are sufficient for detecting asymptomatic metastatic bone lesions. ${ }^{12}$ The author also reported that the sensitivity was $93.3 \%$. 
By different authors, the bone scan sensitivity was found about $62-89 \% .{ }^{2}$ In our study, $77 \%$ patients were found positive for metastases in whole body bone scintigraphy.

\section{Conclusion}

It may be recommended that Tc 99m MDP Bone scintiscanning should be used as the preferred screening investigation because of its low cost, wide availability, usefulness in imaging the entire skeleton and high sensitivity. It can plays a pivotal role for detection of skeletal metastasis, in lung cancer patients in a developing country like Bangladesh and can help the clinicians in the rational approach for management of patient with lung cancers.

\section{References}

1. K. Furugaki, Y. Moriya, T. Iwai , Erlotinib inhibits osteolytic bone invasion of human non-small-cell lung cancer cell line NCI-H292. Clinical and Experimental Metastasis. 2011; 28( 7): 649-59.

2. Krishnamurthy GT, Tubis M, Hiss J, Blahd WH, Distribution pattern of metastatic bone disease. A need for total body skeletal image. JAMA. 1977; 237(23): 2504-06.

3. Aoki J, Inoue T, Tomiyoshi K, Nuclear imaging of bone tumors: FDG-PET. Semin Musculoskelet Radiol 2001; 5(2):183-87.

4. Cook GJ, Fogelman I. Detection of bone metastases in cancer patients by $18 \mathrm{~F}$-fluoride and $18 \mathrm{~F}$ fluorodeoxyglucose positron emission tomography. Q J Nucl Med. 2001; 45 (1):47-52.

5. R. E. Coleman, Metastatic bone disease: clinical features, pathophysiology and treatment strategies. Cancer Treatment Reviews. 2001; 27(3), $165-76$.

6. T. Chu, J. Teng, L. Jiang, H. Zhong, and B. Han, Lung cancer-derived Dickkopf1 is associated with bone metastasis and the mechanism involves the inhibition of osteoblast differentiation. Biochemical and Biophysical Research. 2014; 443(3):962-68.

7. Erturan S,Yaman M, Aydin G, Uzel I, Musellim B, KaynakK, The role of whole body bone scanning and clinical factors in detecting bone metastases in patients with non small cell lung cancer. Chest. 2005; 127(2): 449-51.

8. Robert E Coleman, Clinical Features of Metastatic Bone Disease and Risk of Skeletal Morbidity. Clinical Cancer Research. 2006; (12): 6244-49.

9. Ebert W, Muley T, Herb KP, Schmidt-Gayk H, Comparison of bone scintigraphy with bone markers in the diagnosis of bone metastasis in lung carcinoma patients. Anticancer Res. 2004; (24):3193-01.

10. Iordanidou L, Trivizaki E, Saranti S,Georgakopolos A, Bolanos N, Baltagiannis $\mathrm{N}$ et al. Is there a role of whole body bone scan in early stages of non small cell lung cancer patients? J BUON 2006; 11(4): 491-97.

11. Roodman GD. Mechanisms of disease: mechanisms of bone metastasis. The New England Journal of Medicine. 2004; 350(16). 1655-64.

12. Joo-Won M, Sang-Won U, Jae-Jun Y, Chul-Gyu Y, Han SK, Young-Soo S et al. The Role of WholeBody FDG PET/CT, Tc 99m MDP Bone Scintigraphy, and Serum Alkaline Phosphatase in Detecting Bone Metastasis in Patients with Newly Diagnosed Lung Cancer. J Korean Med Sci. 2009; 24 (2): 275-80. 